This document is the Accepted Manuscript version of a Published Work that appeared in final form in The Journal of Physical Chemistry C, copyright (C) American Chemical Society after peer review and technical editing by the publisher. To access the final edited and published work see http://dx.doi.org/10.1021/jp512328h.

\title{
Free Energy Assessment of Water Structures and Their Dissociation on $\mathrm{Ru}(0001)$
}

\author{
Guillem Revilla-López, ${ }^{\dagger}$ Piotr Błoński, ${ }^{\ddagger}$ and Núria López ${ }^{* \dagger}$ \\ ${ }^{\dagger}$ Institute of Chemical Research of Catalonia, ICIQ Avenida Països Catalans 16, 43007 Tarragona, Spain \\ ${ }^{\ddagger}$ Institute of Nuclear Physics, Polish Academy of Sciences, ulica Radzikowskiego 152, PL-31-342 Krakow, Poland
}

Supporting Information

ABSTRACT: The free energy landscape of the structures and dissociation degree of the first water and heavy water adlayers on $\mathrm{Ru}(0001)$ surface is presented. Thermodynamically favored interconversion routes connecting different experimentally reported structures are suggested based on free energy calculations. On going from low to high water coverage, one-dimensional (1D) periodic chain-like structures with small, or zero, water dissociation degree like Chain-4a motifs are found to be very stable intermediates in the formation of dissociated and molecular ice-like bilayers, respectively. The isotopic effects on the dissociation degree of the ice-like bilayers are estimated: a preference for the halfdissociated form is found for $\mathrm{H}_{2} \mathrm{O}$ at temperatures below $275 \mathrm{~K}$ followed by energetic degeneration between all bilayers dissociated over that threshold. Instead, for heavy water this temperature is shifted to $225 \mathrm{~K}$. Moreover, the configurational entropy due to the different arrangements of dissociated and molecular flat molecules further contributes to set the energies of all these structures within a small energy window that make experimental identification difficult.

\section{INTRODUCTION}

Water adsorption on metal surfaces remains an area of intense research, as it is involved in corrosion, electrochemistry and heterogeneous catalysis. ${ }^{1}$ Many of these studies have focused on the structure of the first water layer adsorbed on $\mathrm{Ru}(0001)$ for which a myriad of apparently unconnected motifs have been reported depending on the experimental conditions.

Initially, the full coverage structure of the water layer on $\mathrm{Ru}(0001)$, coverage $0.67 \mathrm{ML}$, was described as an hexagonal ice-like, $I_{h}$-like Figure 1a. In this structure, the motifs are formed by hexagonal water clusters linked in a honeycomb network with flat and down (up) molecules in a 1:1 proportion. The degree of dissociation was suggested to be variable, ${ }^{2-11}$ Figure $1 \mathrm{~b}$, where some of the down molecules split into hydroxyls and protons that are attached to the surface. The number of hydroxyls in the motifs is still a matter of discussion and can range from half $I_{h}-4 / 8$ of the molecules to 0 , the $I_{h}-0 / 8$ motif, the dissociation degree has been reported to strongly influence the catalytic activity of $\mathrm{Ru}(0001)$ in an aqueous environment. ${ }^{12}$ Both temperature-programmed desorption (TPD) and scanning tunneling microscopy (STM) results were found to be strongly dependent on the dosing temperature and the hydrogen isotope (either protium or deuterium). ${ }^{13-15}$ In addition, the bias voltage in STM could induce further bond cleavages. All these hurdles have rendered the question of the dissociation degree a difficult point to assess experimentally. ${ }^{16}$ In parallel, other isoforms corresponding to the same coverage such as chains, ${ }^{15}$ Figure $1 \mathrm{c}$, were found to coexist under certain conditions. In the chain structure, rows of flat and down molecules are found as connected strips. The main difference from $I_{h}$ is the local coordination of each water

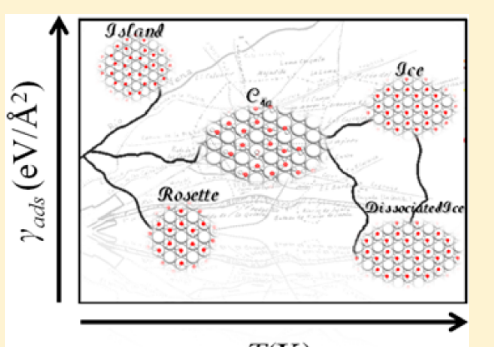

$T(\mathrm{~K})$

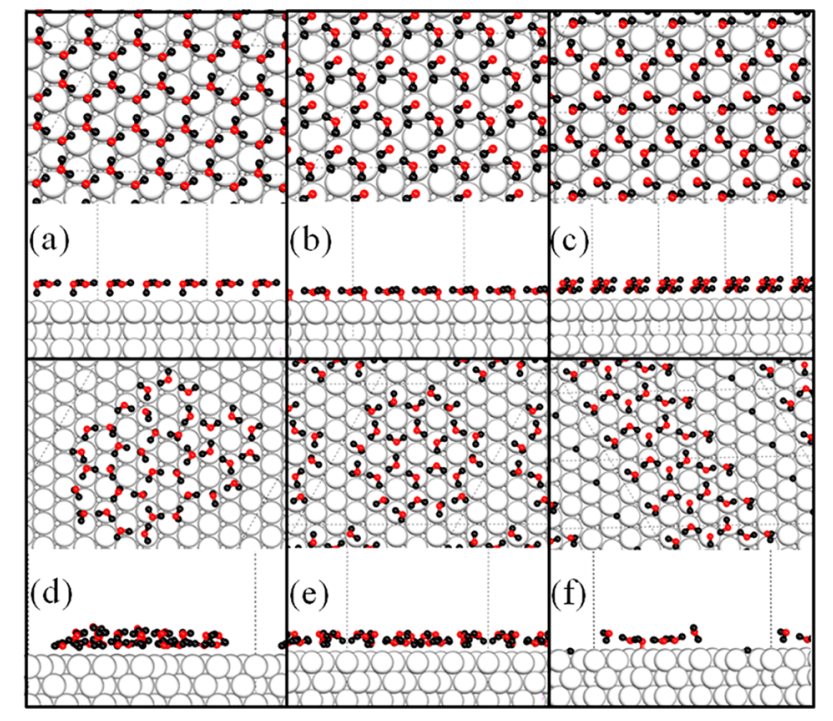

Figure 1. Axial and longitudinal views on $\mathrm{Ru}(0001)$ of (a) $I_{h}-0 / 8$, (b) $I_{h}-4 / 8$, (c) chains; (d) island; (e) rosette, and (f) $\mathrm{C}_{4 \mathrm{a}}-2 / 12$. Oxygen, hydrogen, and ruthenium atoms are represented by red, black, and white spheres, respectively.

molecule. For instance, in chains, flat molecules have in their first coordination sphere at least two flat molecules, a pattern never found in $I_{h}$ structures. 
The interconversion of different motifs and how the complete covering of the surface by increasing quantities of water occurs remains open. Besides, the question remains of how the intermediate coverages turn into either an $I_{h}$ or chain motif. In particular, at low coverages $(0.33 \mathrm{ML})$, isolated clusters in the form of island motifs that combine 10 sixmembered cycles and a five-membered one (Figure 1d) ${ }^{17}$ have been reported. Other islands like the rosette, Figure 1e, present a central flat six-membered ring water core surrounded by six more hexagons. This is the pattern at the core of $I_{h}$ except for the fact that the core presents the coordination motif of the chains (flat molecules are first neighbors to other flat molecules), and it is strongly corrugated, as only the core is in contact with the metal. Recently, Salmeron et al. ${ }^{18}$ via a combined STM-density functional theory (DFT) study have pointed that water bilayer formation on $\mathrm{Ru}(0001)$ might be mediated by a chain-like structure. This new chain motif presents a central strip made of six-membered rings made of four flat water and two dissociated $\mathrm{H}$-down monomers. Two more $\mathrm{H}$-down monomers are adsorbed at two opposite vertexes, thus forming five-remembered uncompleted hexagons at each side of the central strip. In total, this structure contains 12 water molecules, of which 2 are dissociated, $2 / 12$, and there are four rows of bare metal atoms in the $y$ direction between motifs, hereafter referred to as $\mathrm{C}_{4 \mathrm{a}}$ in Figure 1f. The rationale for this central role lays on the one-dimensional (1D) periodicity of the motif in combination with its intermediate coverage, $0.40 \mathrm{ML} .{ }^{19}$ The latter coverage lies in-between that of the ice-like bilayer and the rosette, which presents an exclusion zone for water adsorption around it due to the arrangement of the $\mathrm{H}$-down water monomers in the vertexes.

DFT calculations have provided electronic adsorption energies for most of these motifs at $0 \mathrm{~K}^{20}$ Yet, experiments are performed at finite temperatures for which thermal effects contribute and might rule provided that small energy differences are involved. This work presents the free energy landscape of $\mathrm{H}_{2} \mathrm{O}$ adsorption on $\mathrm{Ru}(0001)$ and the heavy water, $\mathrm{D}_{2} \mathrm{O}$, to assess the energy differences between the structures proposed at intermediate and bilayer coverages.

\section{COMPUTATIONAL DETAILS}

All DFT calculations in this work were carried out with the Vienna $\mathrm{Ab}$ initio Simulation Package (VASP)..$^{21,22}$ The electron-ion interactions were described using the projectoraugmented-wave (PAW) formalism. ${ }^{23,24}$ The plane-wave set contained components with energies up to $400 \mathrm{eV}$, and both energies and structures remain unchanged from this cutoff up to $700 \mathrm{eV}$. The Perdew, Burke, and Ernzerhof (PBE) functional was used for electronic exchange and correlation effects in the generalized-gradient approximation (GGA). ${ }^{25}$ Dispersion energies were accounted for by the semiempirical DFT-D2 ${ }^{26-28}$ approach with the parameters optimized in the group for the $\mathrm{Ru}$ metal surface. ${ }^{29}$ The electronic degrees of freedom were relaxed until the change in total energy between successive iteration steps was below $10^{-6} \mathrm{eV}$. The five layers of the $\mathrm{Ru}$ slab were kept in their bulk-like positions interleaved by $16 \AA$ vacuum and the water molecules were left free to move until the forces acting on them were lower than $25 \mathrm{meV} / \AA^{2}$. The Brillouin zone was sampled using $3 \times 3 \times 1 \Gamma$-centered k-point mesh, and a Methfessel-Paxton ${ }^{30}$ smearing of $0.2 \mathrm{eV}$ was applied. Vibrational modes were obtained within the harmonic approximation by diagonalization of the numeric Hessian matrix obtained by $\pm 0.01 \AA$ displacements and calculated at the
$\Gamma$-point only. $\mathrm{D}_{2} \mathrm{O}$ frequencies were reevaluated after considering the mass of deuterium.

The water/metal interface of $I_{h}-0 / 8$ bilayer was represented by a periodically repeated supercell containing $w=32$ total water molecules, $16 \mathrm{H}$-down and 16 flat, forming a $4 \sqrt{3} 34 \sqrt{3}$ - R30.0 $0^{\circ}$ pattern on 48 metal atoms, $m$ (see Figure 1a). In this structure, intermediate dissociation degrees were built by reassociating water molecules from the half-dissociated that presents a 0.5 dissociation degree, that is, four dissociated $\mathrm{H}$ down momers per each eight total water molecules $(4 / 8$, Figure 1b). Thus, 0 (0/8, $\left.I_{h^{-}} 0 / 8\right), 0.13(1 / 8), 0.25(2 / 8)$, and 0.38 (3/ 8) dissociation degrees were evaluated.

As for the low coverage regime, the island model consisted of $w=32$ and $m=108$, thus rendering a $0.33 \mathrm{ML}$, Figure 1d; chains contain $w=8$ and $m=12,0.67 \mathrm{ML}$ in Figure 1c, and the rosette contains $w=24$ and $m=48,0.5 \mathrm{ML}$ in Figure $1 \mathrm{~d}$. The $\mathrm{C}_{4 \mathrm{a}}$ motif reported by Salmeron et al. ${ }^{18}$ presents $1 \mathrm{D}$ periodicity formed by incomplete chains separated by four rows of bare metal atoms, Figure 1f. $\mathrm{C}_{4 \mathrm{a}}$ contains $w=12$ and $m=30$ with $0.40 \mathrm{ML}$ coverage and two dissociated water molecules, $\mathrm{C}_{4 \mathrm{a}}-2$ / 12; the molecular form of this system, $\mathrm{C}_{4 \mathrm{a}}-0 / 12$, has also been considered in this work.

The adsorption energy, $E_{\text {ads }}$ in $\mathrm{eV} / \mathrm{H}_{2} \mathrm{O}$, is defined with respect to the gas-phase water monomer: $E_{\text {ads }}=E_{\text {sys }}-\left(E_{\text {slab }}+\right.$ $\left.n_{\mathrm{w}} \cdot E_{\mathrm{w}}\right)$, where $E_{\text {sys }}$ is the energy of the metal slab with adsorbates, $E_{\text {slab }}$ is the energy of the bare metal slab, $n_{\mathrm{w}}$ is the number of adsorbed water molecules, and $E_{\mathrm{w}}$ is the energy of an isolated water molecule in the gas-phase. The zero-point vibrational energy of adsorption, $\Delta E_{\text {ads }}^{\mathrm{ZPV}}$, is calculated as $\sum_{i}(h /$ 2) $v_{i}$, where $v_{i}$ stands for the vibrational frequencies and $h$ is the Planck constant. All structures were confirmed as real minima; no imaginary vibrational modes were found. Of the $3 \mathrm{~N}-6$ vibrations, the very low energy modes would be better represented as frustrated rotations as demonstrated by van Santen et al. ${ }^{31}$ Following common practice in molecular studies and to avoid the spurious contributions of thermal and entropic contributions of these low energy modes when treated with standard vibrational partition functions, we removed all the frequencies below $100 \mathrm{~cm}^{-1}$. The free energy of adsorption, $G_{\text {ads }}$ was then calculated according to $G_{\text {ads }}=G_{\text {sys }}-\left(G_{\text {slab }}+\right.$ $\left.n_{\mathrm{w}} \cdot \mu_{\mathrm{H}_{2} \mathrm{O}}\right)$, where $\mu_{\mathrm{H}_{2} \mathrm{O}}$ is the chemical potential of water per molecule at 1 bar pressure and a given temperature, and $G_{\text {sys }}$ is the free energy of the system according to $G_{\text {sys }} \approx H_{\text {sys }}^{\text {corr }}-T S_{\text {sys }}^{\text {vib }}$; $H_{\text {sys }}^{\text {corr }}=E_{\text {tot }}^{\text {sys }}+k_{\mathrm{B}} T$. T and $k_{\mathrm{B}}$ stand for temperature and Boltzmann constant. The methodology follows that in ref 32 .

For partially dissociated structures, the different distributions between molecular and dissociated molecules can present different configurations, leading to a configurational entropy in terms of free energy, $G_{\text {sys }} \approx H_{\text {sys }}^{\text {corr }}-T S_{\text {sys }}^{\text {vib }}-T S_{\text {conf }}$ In our case, this has only been assessed for the complete $I_{h}$ structures as follows: $S_{\text {conf }}=-k_{\mathrm{B}} \ln \Omega$, where $\Omega=\left(\begin{array}{c}w_{\mathrm{dw}} \\ w_{\mathrm{diss}}\end{array}\right)=\frac{w_{\mathrm{dw}} !}{\left(w_{\mathrm{dw}}-w_{\mathrm{diss}}\right) ! w_{\mathrm{diss}} !}$, where $w_{\mathrm{dw}}$ stands for the number of $\mathrm{H}$-down water monomers in the ensembles and $w_{\text {diss }}$ accounts for the dissociated ones within this set. For the $I_{h}$ structures, we are reporting the system surface energies, $\gamma_{\text {sys }}=G_{\text {sys }} / A .^{33}$

\section{RESULTS AND DISCUSSION}

Cohesive energy in three-dimensional (3D) hexagonal ice, $I_{h}$, has traditionally been taken as the energy threshold for adlayer stability on metals. ${ }^{34}$ The cohesive energy values, $\Delta E_{\mathrm{ads}}^{\mathrm{ZPV}}$, according to our calculations is $-0.54 \mathrm{eV} / \mathrm{H}_{2} \mathrm{O}$, and the 


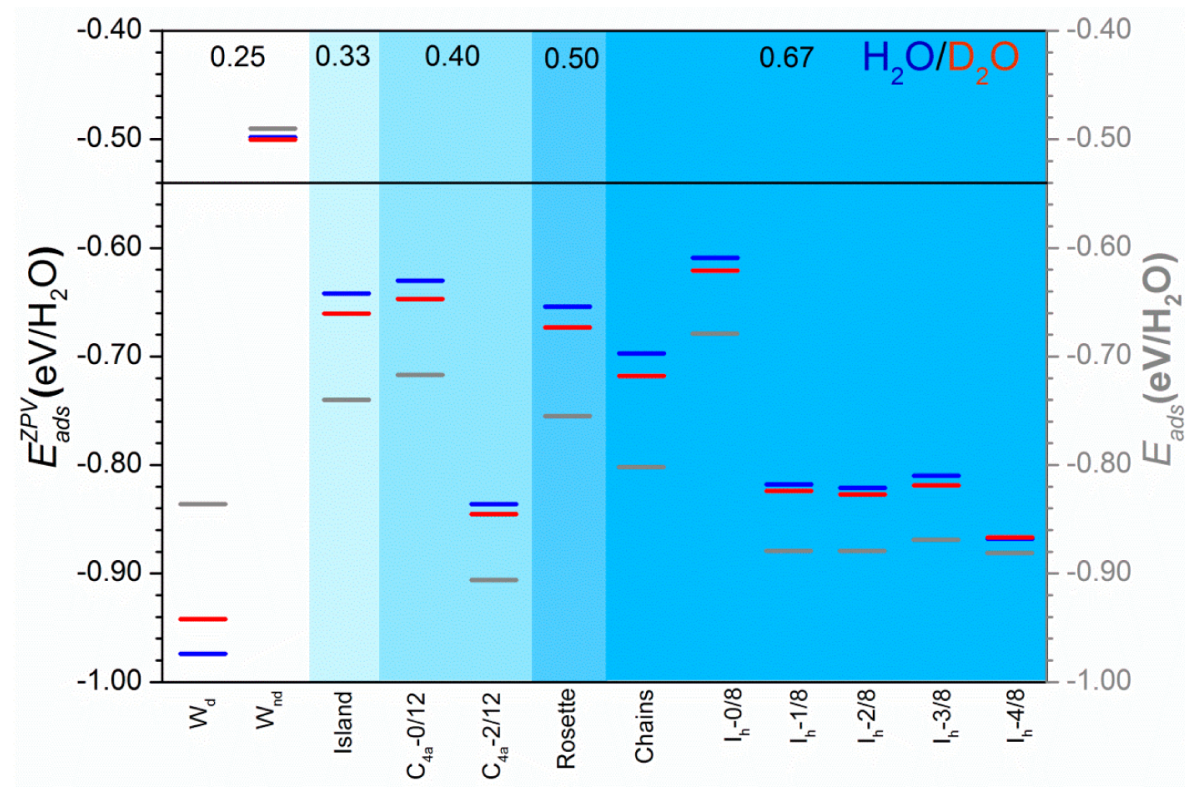

Figure 2. $E_{\text {ads }}$ in $\mathrm{eV} \cdot \mathrm{H}_{2} \mathrm{O}^{-1}$ is presented (gray marks right scale). $E_{\text {ads }}^{\mathrm{ZPV}}$ in $\mathrm{eV} \cdot \mathrm{H}_{2} \mathrm{O}^{-1}$ (black scale left) for the adsorption of $\mathrm{H}_{2} \mathrm{O}\left(\right.$ blue) and $\mathrm{D}_{2} \mathrm{O}$ (red). For each ensemble, the coverage is described at the top in ML. Systems: $\mathrm{W}_{\mathrm{d}}$ isolated dissociated water; $\mathrm{W}_{\text {nd }}$ molecular water; island; the chains reported by Salmeron et al.: ${ }^{18} \mathrm{C}_{4 \mathrm{a}}-2 / 12$ and $\mathrm{C}_{4 \mathrm{a}} \mathrm{-}-12$; rosette; chains and different $I_{h}$-d/total models with different amount of dissociated (d) molecules. The black line is the $3 \mathrm{D}$ ice $E_{\mathrm{ads}}^{\mathrm{ZPV}}$. All energies are in $\mathrm{eV} / \mathrm{H}_{2} \mathrm{O}$. The equivalent in terms of interaction energy per surface is presented in Supporting Information Figure S1.

experimental value is $-0.61 \mathrm{eV} / \mathrm{H}_{2} \mathrm{O}^{5}$ In key places of our figures, comparisons to the cohesive energy of bulk ice are presented to simplify the comparison to the literature. ${ }^{34}$ However, we have preferred to present water-normalized adsorption energies: $E_{\mathrm{ads}}$ and $E_{\mathrm{ads}}^{\mathrm{ZPV}}$ as in Figure 2.

The larger stability of the dissociated single $\mathrm{H}_{2} \mathrm{O}$ adsorption, $E_{\text {ads }}^{\mathrm{ZPV}}$ Figure 2, over the molecular one agrees with previous estimates by Michaelides et al. $^{7}$ Moreover, dissociative adsorption is far more stable than any other water molecule belonging to an ensemble. In addition, dissociated $\mathrm{H}_{2} \mathrm{O}$ adsorption is stabilized over $\mathrm{D}_{2} \mathrm{O}$, which is opposite to the observed tendency for the rest of ensembles. This change is related to the lower mass of protium, which makes the vibrational frequencies of split $\mathrm{H}$ and $\mathrm{OH}$ higher than for $\mathrm{D}$ and DO. As coverage increases, the island motif ( $0.33 \mathrm{ML}$, Figure 1d) shows up, forming a noncontinuous overlayer with multimembered cycles. In terms of energy per molecule, the island motif is metastable with respect to the $\mathrm{C}_{4 a}-2 / 12$ by 166 $\mathrm{meV} / \mathrm{H}_{2} \mathrm{O}$, while it is virtually isoenergetic, $E_{\mathrm{ads}}^{\mathrm{ZPV}}=-0.740 \mathrm{eV} /$ $\mathrm{H}_{2} \mathrm{O}$; with the rosette, $-0.755 \mathrm{eV} / \mathrm{H}_{2} \mathrm{O},{ }^{19,34}$ and with the $\mathrm{C}_{4 \mathrm{a}^{-}}$ $0 / 12,-0.717 \mathrm{eV} / \mathrm{H}_{2} \mathrm{O} . \mathrm{C}_{4 \mathrm{a}}-0 / 12$ can develop exothermically, by simple water addition and a few rearrangements, into chains. The difference in the adsorption energies is rather small $\Delta E_{\mathrm{ads}}^{\mathrm{ZPV}}$ $=-67 \mathrm{meV} / \mathrm{H}_{2} \mathrm{O}$ obviously, the total energy is lower for the system with more molecules. Moreover, chains can evolve into $I_{h}-0 / 8$ bilayer by a few rearrangements, but the $I_{h}$ is less stable by $87 \mathrm{meV} / \mathrm{H}_{2} \mathrm{O}$. Instead there might exist a direct path that relates $\mathrm{C}_{4 \mathrm{a}}-0 / 12$ to $I_{h^{-}}-0 / 8$ as it implies fewer molecular rearrangements, only 1 in 6 water monomers, and the energies per molecule are closer $\left(21 \mathrm{meV} / \mathrm{H}_{2} \mathrm{O}\right)$. In parallel, the formation of partially dissociated $I_{h}$-like bilayers could be based on $\mathrm{C}_{4 \mathrm{a}}-2 / 12$. This structure is virtually isoenergetic in normalized energies with $I_{h}-2 / 8, \Delta E_{\text {ads }}^{\mathrm{ZPV}}=15 \mathrm{meV} / \mathrm{H}_{2} \mathrm{O}$. Moreover, partially dissociated $I_{h}$ isoforms seem energetically degenerate, all lay within $11 \mathrm{meV} / \mathrm{H}_{2} \mathrm{O}$, and metastable with the half-dissociated form by $50 \mathrm{meV} / \mathrm{H}_{2} \mathrm{O}$, which opens the thermodynamic possibility to rapid interconversion between these structures. $^{35}$

Heavy water (Figure 2) slightly alters the relative energy scales discussed for water. The energy difference from $\mathrm{C}_{4 \mathrm{a}}-0 / 12$ to chains remains unchanged at $-67 \mathrm{meV} / \mathrm{D}_{2} \mathrm{O}$ in sharp contrast with the Chains to $I_{h}-0 / 8$ at $103 \mathrm{meV} / \mathrm{D}_{2} \mathrm{O}$. Conversely, the $\mathrm{C}_{4 \mathrm{a}}-0 / 12$ to $\mathrm{I}_{h}-0 / 8$ unbalance increases to 27 $\mathrm{meV} / \mathrm{D}_{2} \mathrm{O}$, only $6 \mathrm{meV}$ more than the values corresponding to $\mathrm{H}_{2} \mathrm{O}$. The energy difference between $I_{h}-2 / 8$ and $\mathrm{C}_{4 \mathrm{a}}-2 / 12$ is similar to $\mathrm{H}_{2} \mathrm{O}, 18 \mathrm{meV} / \mathrm{D}_{2} \mathrm{O}$. The most important point, though, concerns the energy span for the partially dissociated $I_{h}$-like forms that are reduced to $5 \mathrm{meV} / \mathrm{D}_{2} \mathrm{O}$ for heavy water. $\mathrm{D}_{2} \mathrm{O}$ also tightens the gap between all the $I_{h}$-like bilayers: the gap between $I_{h}-0 / 8$ and $I_{h}-4 / 8$ is reduced by 13 to $246 \mathrm{meV} /$ $\mathrm{D}_{2} \mathrm{O}$, and a similar reduction, $11 \mathrm{meV}$, is observed between $I_{h^{-}}$ $4 / 8$ and the other partially dissociated configurations.

Figure 3 shows the free energy of adsorption, $G_{\mathrm{ads}}$, in the temperature range between 125 and $300 \mathrm{~K}$ for all continuous $\mathrm{H}_{2} \mathrm{O}$ (panel a) and $\mathrm{D}_{2} \mathrm{O}$ (panel b) motifs. Surface-normalized energies are reported in Figure $\mathrm{S} 2$ in the Supporting Information. The energy ranking in terms of $E_{\text {ads }}^{\mathrm{ZPV}}$ for $\mathrm{H}_{2} \mathrm{O}$ is mostly maintained when temperature effects are included. As can be seen, $\mathrm{C}_{4 a}-0 / 12$ (Figure $3 \mathrm{a}$ ) is more stable than the $I_{h}-0 / 8$ bilayer by $44 \mathrm{meV} / \mathrm{H}_{2} \mathrm{O}$. On the other hand, $\mathrm{C}_{4 \mathrm{a}}-2 / 12$ is isoenergetic with the partially dissociated $I_{h}$-like bilayers and metastable with $I_{h}-4 / 8$ up to $225 \mathrm{~K}$. Over this temperature, the ranking is turned upside down, and $\mathrm{C}_{4 \mathrm{a}}-2 / 12$ becomes the lowest energy structure as $I_{h}-4 / 8$ and $I_{h}-2 / 8$ are slightly disfavored, by 10 and $15 \mathrm{meV} / \mathrm{H}_{2} \mathrm{O}$, respectively. Further on, all the partially dissociated forms and $\mathrm{C}_{4 \mathrm{a}}-2 / 12$ are within an energy span of $39 \mathrm{meV} / \mathrm{H}_{2} \mathrm{O}$. $I_{h}-4 / 8$ bilayer is always more stable than the partially dissociated forms and the $I_{h}-0 / 8$ below $275 \mathrm{~K}$, whereas over this temperature all dissociated structures become degenerate. The differential stabilization found for $\mathrm{D}_{2} \mathrm{O}$ in terms of $E_{\text {ads }}^{\mathrm{ZPV}}$ is also reproduced by $G_{\mathrm{ads}}$, Figure $3 \mathrm{~b}$. Throughout the temperature range, the adsorption energy of 


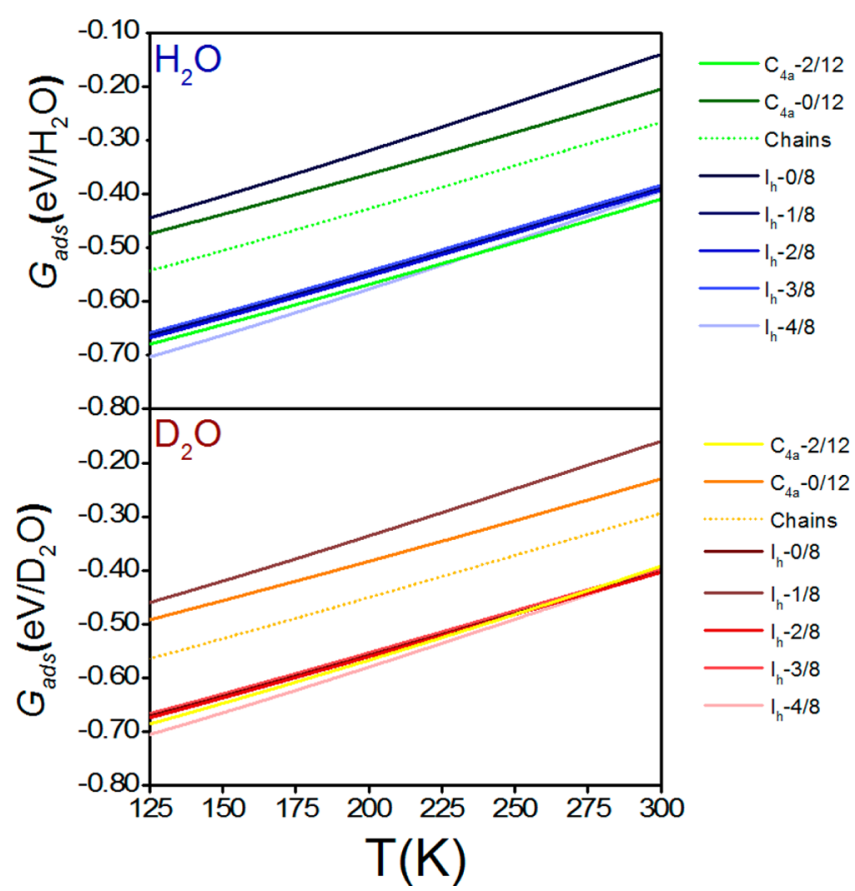

Figure 3. $\mathrm{G}_{\text {ads }}$ in $\mathrm{eV} / \mathrm{H}_{2} \mathrm{O}$ and $\mathrm{eV} / \mathrm{D}_{2} \mathrm{O}$, as a function of temperature for the different ensembles in the present work.

$\mathrm{C}_{4 \mathrm{a}}-0 / 12$ is more exothermic than that of $I_{h}$-like bilayer by 47 $\mathrm{eV} / \mathrm{D}_{2} \mathrm{O}$, whereas all dissociated forms of $I_{h}$-like bilayers and $\mathrm{C}_{4 \mathrm{a}}-2 / 12$ are within a range of $30 \mathrm{meV} / \mathrm{D}_{2} \mathrm{O} . I_{h}-4 / 8$ presents the lowest adsorption energy below $225 \mathrm{~K}$, with $\mathrm{C}_{4 \mathrm{a}}-2 / 12$ and $I_{h}-2 / 8$ being disfavored by 17 and $30 \mathrm{meV} / \mathrm{D}_{2} \mathrm{O}$, respectively. Over $225 \mathrm{~K}$, the same energy degeneration found for $\mathrm{H}_{2} \mathrm{O}$ over
$275 \mathrm{~K}$, but including $\mathrm{C}_{4 \mathrm{a}}-2 / 12$, is reproduced since previous gaps are reduced to 8 and $2 \mathrm{meV} / \mathrm{D}_{2} \mathrm{O}$, respectively.

Figure 4 presents the surface normalized free energy differences, $\Delta \gamma$, of the dissociated bilayers from the molecular form in $\mathrm{meV} \cdot \AA^{-2}$. The overall energy for the complete ensemble for 32 molecules is presented here. In Figure $4 a, c$, the total energy is always favorable to the $I_{h}-4 / 8$ and after (Figure $4 \mathrm{~b}, \mathrm{~d}$ ), considering the configurational entropy. The number of configurations for the dissociated and nondissociated structures, $\Omega$, peaks for the partially dissociated $I_{h}-2 / 8$ structure being 1 for the two extremes: $I_{h}-0 / 8$ and $I_{h}-4 / 8$. The entropic contribution leads to a stabilization of $I_{h}-2 / 8$ over $I_{h}-1 / 8$ and $I_{h}-3 / 8$ throughout the range. Yet, $I_{h}-4 / 8$ remains the lowest energy isoform within the considered finite temperatures. A virtual energy degeneration is detected for $\mathrm{H}_{2} \mathrm{O}$ over $250 \mathrm{~K}$ and for $\mathrm{D}_{2} \mathrm{O}$ over $220 \mathrm{~K}$ since all dissociated ensembles are within a span of 3 and $\mathrm{meV} \cdot \AA^{-2}$. The thermodynamic degeneration form can explain the different degree of dissociation reported in the experiments. Moreover, as the energy differences are small, dynamic behavior is expected. Thus, the highly variable dissociation rate found in the experiments is explained not only by the thermal contributions but also by the configurational component.

The work function, $\phi$, is a widely used experimental technique employed to characterize water adlayers on metals. Changes in the work function can be attributed to changes in the dipole moment of adsorbates on the surface, ${ }^{36-38}$ which in polyatomic molecules is related to the orientation of molecules with regard to the surface, the coverage, and the structure of the ensemble formed by them. The values for the systems studied in this work have been calculated by subtracting the Fermi level to the average electrostatic potential energy at the half height of the $z$ direction, right in the vacuum of the slab system. These

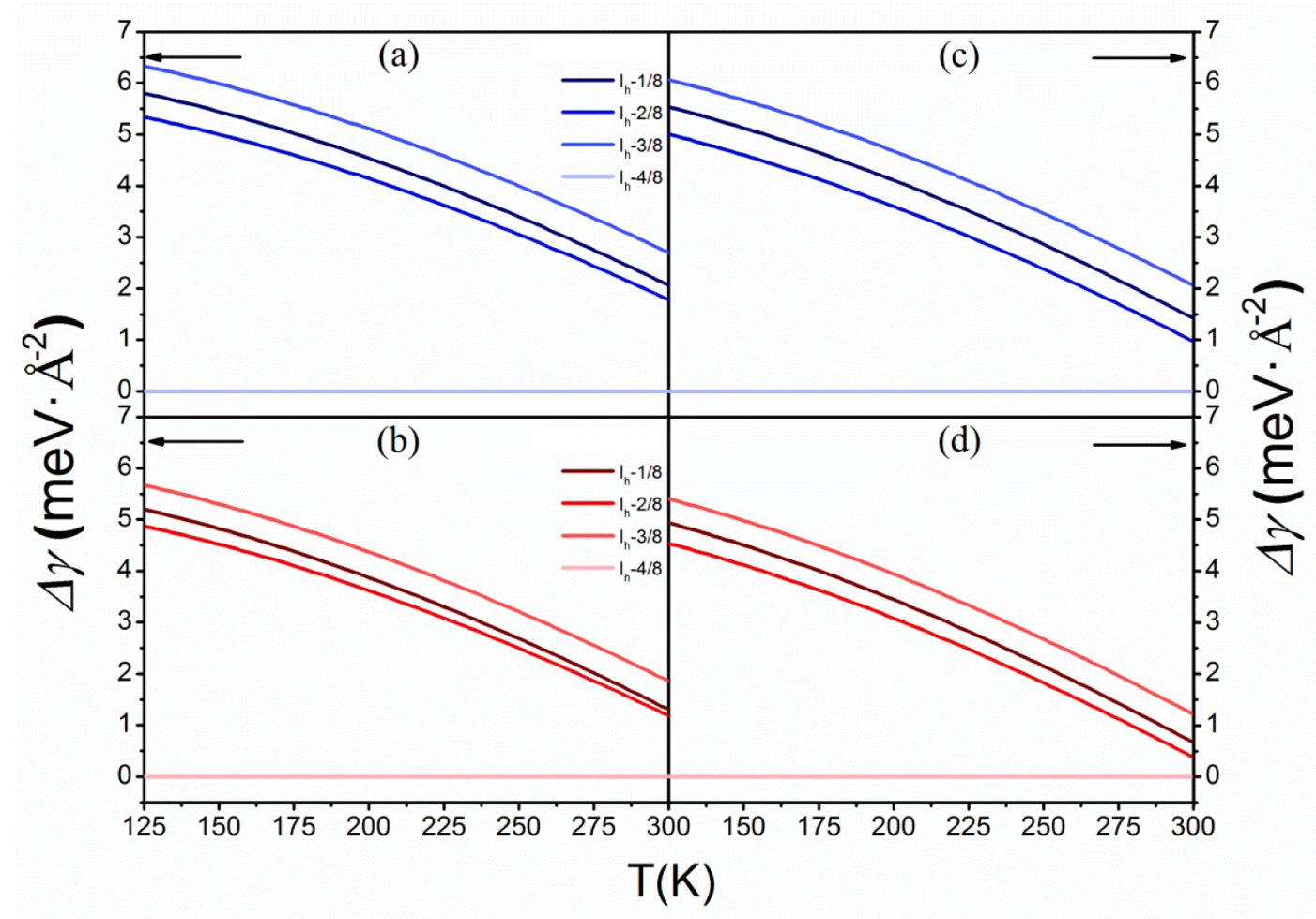

Figure 4. Surface adsorption energy relative to the $I_{h}-4 / 8$ system, $\Delta \gamma$, in $m e V \cdot \AA^{-2}$, of the dissociated bilayers as a function of temperature for $\mathrm{H}_{2} \mathrm{O}$ $(\mathrm{a}, \mathrm{c})$ and $\mathrm{D}_{2} \mathrm{O}(\mathrm{b}, \mathrm{d})$. Energies without $(\mathrm{a}, \mathrm{b})$ and with $(\mathrm{c}, \mathrm{d})$ configurational entropy contributions. 
measurements reveal relevant differences between both the lowest and highest coverage motifs and slight gaps between the molecular and dissociated states of the ice-bilayer, 4.64 and 4.89 $\mathrm{eV}$, respectively. These values represent -0.31 and $-0.06 \mathrm{eV}$ variation of the work function, $\Delta \phi$, with respect tothat of the bare $\mathrm{Ru}(0001)$ surface, $4.95 \mathrm{eV}$. That is in nice agreement with previous theoretical calculations by Schnur and Gro $\beta .^{36}$ However, when the values for the partially dissociated bilayers are regarded, $4.84,4.86$, and 4.89 for the $1 / 8,2 / 8$ and $3 / 8$ dissociated forms, respectively, the differences with $I_{h}-4 / 8$ fade away, while those with the molecular form are maintained. In addition, island and rosette motifs present values of 4.73 and $4.64 \mathrm{eV}$, whereas chains and the $\mathrm{C}_{4 \mathrm{a}}-0 / 12$ and $\mathrm{C}_{4 \mathrm{a}}-2 / 12$ present $4.67,4.67$, and $4.72 \mathrm{eV}$, respectively. These results are in line with the value for the molecular $I_{h}-0 / 8$ bilayer, which suggests that work function by itself is partially useful to discern the dissociation state, as previously reported, ${ }^{3-5}$ and hardly useful for discriminating between different coverage regimes.

\section{CONCLUSIONS}

In conclusion, the free energy landscape of experimentally reported $\mathrm{H}_{2} \mathrm{O}$ motifs on $\mathrm{Ru}(0001)$ has been described. $E_{\text {ads }}^{\mathrm{ZPV}}$ points at the key role of $\mathrm{C}_{4 a}-0 / 12$ and $\mathrm{C}_{4 \mathrm{a}}-2 / 12$ in the formation of $I_{h}-0 / 8$ bilayer and its dissociated forms, respectively. The latter is unaffected by the use of $\mathrm{H}_{2} \mathrm{O}$ or $\mathrm{D}_{2} \mathrm{O}$. Thus, the importance of bilayer forming pathways through isolated two-dimensional (2D) clusters like the rosette and island has been downgraded to enhance those through chain-like $1 \mathrm{D}$ periodic structures. $\mathrm{D}_{2} \mathrm{O}$ induces a stabilization of the partially dissociated $I_{h}$-like bilayers by making them isoenergetic with $I_{h}-4 / 8$ over $225 \mathrm{~K}$ in sharp contrast with $\mathrm{H}_{2} \mathrm{O}$, for which $\mathrm{I}_{h}-4 / 8$ is preferred until $275 \mathrm{~K}$. Configurational entropy differentially stabilizes the partially dissociated states of the $I_{h}$-like bilayer over $I_{h}-4 / 8$ because of the higher number of feasible configurations for intermediate dissociative states. The latter, combined with the energy closeness of partially dissociated systems, is the cause of the high interconversion rates between structures and the variable observed dissociation degree. Finally, the work function has been found to be a descriptor of the dissociation state of the aqueous adlayer, but not for discerning between intermediate coverages and the structure of the motif formed by water.

\section{ASSOCIATED CONTENT}

\section{S Supporting Information}

$\mathrm{XYZ}$ coordinates and energies of systems employed in this work and rescaled versions of Figures 2 and 3.

\section{AUTHOR INFORMATION}

\section{Corresponding Author}

*E-mail: nlopez@iciq.es.

\section{Notes}

The authors declare no competing financial interest.

\section{ACKNOWLEDGMENTS}

This work has been supported through the ERC Starting Grant: Biomass to Chemicals: Catalysis design for sustainable chemical industry-Theoretical Simulations (ERC-2010-StG-258406). The simulations have been done in the supercomputer MareNostrum at Barcelona Supercomputing Center - Centro Nacional de Supercomputación (The Spanish National Super- computing Center). We thank Dr. Max Garcia-Melchor, Dr. Luca Bellarosa and Msc. Rodrigo García-Muelas for fruitful discussion of vibrational modes and free energy calculations.

\section{REFERENCES}

(1) Carrasco, J.; Hodgson, A.; Michaelides, A. A Molecular Perspective of Water at Metal Interfaces. Nat. Mater. 2012, 11, 667-674.

(2) Doering, D. L.; Madey, T. E. The Adsorption of Water on Clean and Oxygen-Dosed $\mathrm{Ru}(001)$. Surf. Sci. 1982, 123, 305-337.

(3) Thiel, P. A.; Madey, T. E. The Interaction of Water with SolidSurfaces - Fundamental-Aspects. Surf. Sci. Rep. 1987, 7, 211-385.

(4) Held, G.; Menzel, D. Structural Rearrangement by Coadsorption - A LEED-IV Determination of the $\mathrm{Ru}(001)-\mathrm{P}(2 \times 2)(2 \mathrm{O}+\mathrm{CO})$ Structure. Surf. Sci. 1994, 317, 131-142.

(5) Feibelman, P. J. Partial Dissociation of Water on $\mathrm{Ru}(0001)$. Science 2002, 295, 99-102.

(6) Weissenrieder, J.; Mikkelsen, A.; Andersen, J. N.; Feibelman, P. J.; Held, G. Experimental Evidence for a Partially Dissociated Water Bilayer on $\mathrm{Ru}\{0001\}$. Phys. Rev. Lett. 2004, 93, 196102.

(7) Michaelides, A.; Alavi, A.; King, D. A. Different Surface Chemistries of Water on $\mathrm{Ru}\{0001\}$ : From Monomer Adsorption to Partially Dissociated Bilayers. J. Am. Chem. Soc. 2003, 125, 2746-2755.

(8) Michaelides, A.; Alavi, A.; King, D. A. Insight into $\mathrm{H}_{2} \mathrm{O}$-ice Adsorption and Dissociation on Metal Surfaces from First-Principles Simulations. Phys. Rev. B 2004, 69, 113404.

(9) Meng, S.; Wang, E. G.; Gao, S. W. Water Adsorption on Metal Surfaces: A General Picture from Density Functional Theory Studies. Phys. Rev. B 2004, 69, 195404.

(10) Denzler, D. N.; Hess, Ch.; Dudek, R.; Wagner, S.; Frischkorn, Ch.; Wolf, M.; Ertl, G. Interfacial Structure of Water on $\mathrm{Ru}(001)$ Investigated by Vibrational Spectroscopy. Chem. Phys. Lett. 2003, 376, 618.

(11) Feibelman, P. J. What the Stretch Frequency Spectrum of $\mathrm{D}_{2} \mathrm{O} /$ $\mathrm{Ru}(0001)$ Does and Does Not Mean. Chem. Phys. Lett. 2004, 389, $92-$ 95.

(12) García-Muelas, R.; Li, Q; López, N. A Density Functional Theory Comparison of Methanol Decomposition and Reverse Reactions on Metal Surfaces. ACS Catal. 2015, 5, 1027-1036.

(13) Puisto, S. R.; Lerotholi, T. J.; Held, G.; Menzel, D. A Refined LEED Analysis of Water on Ru\{0001\}: An Experimental Test of the Partial Dissociation Model. Surf. Rev. Lett. 2003, 10, 487.

(14) Gallagher, M.; Omer, A.; Darling, G. R.; Hodgson, A. Order and Disorder in the Wetting Layer on $\mathrm{Ru}(0001)$. Faraday Discuss. 2009, 141, 231-249.

(15) Haq, S.; Clay, C.; Darling, G. R.; Zimbitas, G.; Hodgson, A. Growth of Intact Water Ice on $\mathrm{Ru}(0001)$ between 140 and 160K: Experiment and Density-Functional Theory Calculations. Phys. Rev. B 2006, 73, 115414.

(16) Tatarkhanov, M.; Fomin, E.; Salmeron, M.; Andersson, K.; Ogasawara, H.; Petterson, L. G. M.; Nilsson, A.; Cerdá, J. I. The Structure of Mixed $\mathrm{H}_{2} \mathrm{O}-\mathrm{OH}$ Monolayer Films on $\mathrm{Ru}(0001)$. J. Chem. Phys. 2008, 129, 154109.

(17) Maier, S.; Stass, I.; Mitsui, T.; Feibelman, P. J.; Thurmer, K.; Salmeron, M. Adsorbed Water-Molecule Hexagons with Unexpected Rotations in Islands on $\operatorname{Ru}(0001)$ and $\operatorname{Pd}(111)$. Phys. Rev. B 2012, 85, 155434.

(18) Maier, S.; Stass, I.; Cerdá, J. I.; Salmeron, M. Unveiling the Mechanism of Water Partial Dissociation on $\mathrm{Ru}(0001)$. Phys. Rev. Lett. 2014, 112, 126101.

(19) Cerdá, J.; Michaelides, M.; Bocquet, M.-L.; Feibelman, P. J.; Mitsui, T.; Rose, M.; Fomin, E.; Salmeron, M. Novel Water Overlayer Growth on $\operatorname{Pd}(111)$ Characterized with Scanning Tunneling Microscopy and Density Functional Theory. Phys. Rev. Lett. 2004, 93, 116101

(20) Revilla-López, G.; López, N. A Unified Study for Water Adsorption on Metals: Meaningful Models from Structural Motifs. Phys. Chem. Chem. Phys. 2014, 16, 18933-18940. 
(21) Kresse, G.; Furthmüller, J. Efficiency of Ab-Initio Total Energy Calculations for Metals and Semiconductors Using a Plane-Wave Basis Set. Comput. Mater. Sci. 1996, 6, 15-50.

(22) Kresse, G.; Furthmüller, J. Ab initio Calculations of the Cohesive, Elastic, and Dynamical Properties of $\mathrm{CoSi}_{2}$ by Pseudopotential and All-Electron Techniques. Phys. Rev. B 1996, 54, 11169.

(23) Kresse, G.; Joubert, D. From Ultrasoft Pseudopotentials to the Projector Augmented-Wave Method. Phys. Rev. B 1999, 59, 1758.

(24) Blöchl, P. E. Projector Augmented-Wave Method. Phys. Rev. B 1994, 50, 17953.

(25) Perdew, J. P.; Burke, K.; Ernzerhof, K. Generalized Gradient Approximation Made Simple. Phys. Rev. Lett. 1996, 77, 3865.

(26) Hamada, I.; Otani, M. Comparative van der Waals DensityFunctional Study of Graphene on Metal Surfaces. Phys. Rev. B 2010, 82,153412 .

(27) Grimme, S.; Antony, J.; Ehrlich, S.; Krief, H. J. A Consistent and Accurate $A b$ Initio Parametrization of Density Functional Dispersion Correction (DFT-D) for the 94 Elements H-Pu. Chem. Phys. 2010, 132, 154104.

(28) Búcko, T.; Hafner, J.; Lebegue, S.; Angyan, J. G. Improved Description of the Structure of Molecular and Layered Crystals: Ab Initio DFT Calculations with van der Waals Corrections. J. Phys. Chem. A 2010, 114, 11814-11824.

(29) Almora-Barrios, N.; Carchini, G.; Blonski, P.; López, N. Costless Derivation of Dispersion Coefficients for Metal Surfaces. J. Chem. Theory Comput. 2014, 10, 5002-5009.

(30) Methfessel, M.; Paxton, A. T. High-Precision Sampling for Brillouin-Zone Integration in Metals. Phys. Rev. B 1989, 40, 3616

(31) Offermans, W. K.; Jansen, A. P. J.; van Santen, R. A. Ammonia Activation on Platinum $\{111\}$ : A Density Functional Theory Study. Surf. Sci. 2006, 600, 1714-1724.

(32) Bollinger, M. V.; Jacobsen, K. W.; Nørskov, J. K. Atomic and Electronic Structure of $\mathrm{MoS}_{2}$ Nanoparticles. Phys. Rev. B 2003, 67, 085410.

(33) Reuter, K.; Scheffler, M. Composition, Structure and Stability of $\mathrm{RuO}_{2}(110)$ as a Function of Oxygen Pressure. Phys. Rev. B 2001, 65, 035406.

(34) Carrasco, J.; Santra, B.; Klimes, J.; Michaelides, A. To Wet or Not to Wet? Dispersion Forces Tip the Balance for Water Ice on Metal. Phys. Rev. Lett. 2011, 106, 026101.

(35) Lespes, N.; Filhol, J.-S. Using the Electrochemical Dimension to Build Water $/ \mathrm{Ru}(0001)$ Phase Diagram. Surf. Sci. 2015, 631, 8-16.

(36) Schnur, S.; Gro $\beta$, A. Properties of Metal-Water Interfaces Studied from First Principles. New J. Phys. 2009, 11, 125003.

(37) Filhol, J.-S.; Bocquet, M.-L. Charge Control of the Water Monolayer/Pd Interface. Chem. Phys. Lett. 2007, 438, 203-207.

(38) Roman, T.; Gro $\beta$, A. Periodic Density-Functional Calculations on Work-Function Change Induced by Adsorption of Halogens on $\mathrm{Cu}(111)$. Phys. Rev. Lett. 2013, 110, 156804. 